\title{
Educación para la Paz: Un Espacio de Innovación e Intercambio de Buenas Prácticas Docentes
}

\section{Education for Peace: A Space of Innovation and Exchange of Good Teaching Practices}

\author{
Alena Kárpava ${ }^{1 *}$ \\ Verónica Juárez Ramos ${ }^{2}$ \\ ${ }^{1}$ Universidad de Granada, España \\ ${ }^{2}$ Universidad de Extremadura, España
}

\begin{abstract}
Durante el año académico 2018-2019 en la Universidad de Granada se llevó a cabo un Proyecto de Innovación y Buenas Prácticas Docentes que pretendía acercar a los docentes en formación a las vivencias teórico-prácticas de la Educación para la Paz Social, Interna y Gaia. Aplicamos método del aprendizaje basado en problemas, fomentando la observación, experimentación y acción colaborativa con ayuda de las técnicas de la Pedagogía de Freinet y de la Educación Emocional-Transpersonal. La metodología paidocéntrica permitió cumplir con los objetivos del proyecto, construyendo los aprendizajes teóricos de la Educación para la Paz a partir de la vivencia práctica. La evaluación de la mejora docente tenía carácter comprensivo, aplicando como instrumentos el informe final del aprendizaje basado en problemas, el Diario de Paz y las sesiones dialogadas. Como resultado el alumnado fue dotado de herramientas de reflexión crítica y de técnicas de Educación para la Paz, que permitieron incrementar la atención, motivación, participación, autoobservación y autorrealización. Se profundizó en la necesidad de la formación en la Educación para la Paz. Se colaboró con el Movimiento Cooperativo de la Escuela Popular en creación del espacio virtual en la Biblioteca de la Universidad dedicado a la recopilación del material teórico-práctico de la Pedagogía de Freinet.
\end{abstract}

Descriptores: Educación; Paz; Docentes; Universidad; Formación.

During the 2018-2019 academic year at the University of Granada, a Project of Innovation and Good Teaching Practices was carried out due to want to bring teachers in practices, the theoretical-practical experiences of Education for Social, Internal and Gaia Peace. We apply problem-based learning method, encouraging observation, experimentation and collaborative action with the help of Freinet Pedagogy and Emotional-Transpersonal Education techniques. The paidocentric methodology allowed to fulfill the objectives of the project, building the theoretical learning of the Education for Peace from the practical experience. The evaluation of teacher improvement was comprehensive, applying as instruments, the final report of problem-based learning, the Journal of Peace and the dialogue sessions. As a result, students were provided with critical reflection tools and Peace Education techniques, which allowed increasing attention, motivation, participation, selfobservation and self-realization. The need for training in the EpP was deepened. It was collaborated with the Cooperative Movement of the Popular School in creation of the virtual space in the University Library that collects the theoretical and practical material of the Freinet Pedagogy.

Keywords: Education; Peace; Teachers; University; Training.

*Contacto: akarpava@ugr.es

ISSN: 2254-3139

www.rinace.net/riejs/

revistas.uam.es/riejs
Recibido: $\quad 10$ de octubre 2019

$1^{\mathrm{a}}$ Evaluación: 15 de enero 2020

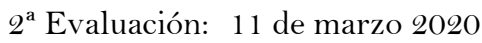

Aceptado: 16 de abril 2020 


\section{Introducción}

En el año académico 2018-2019 se realizó un Proyecto de Innovación y Buenas Prácticas Docentes $(\mathrm{PIBD})^{1}$ que pretendía acercar a los docentes en formación a las vivencias teórico-prácticas de la Educación para la Paz (EpP). En los dos primeros apartados del presente artículo hacemos una introducción teórica a la EpP. Los apartados tres y cuatro integran la justificación normativa y psicopedagógica de la EpP. En el quinto describimos brevemente el proyecto de EpP como espacio de innovación docente.

La idea del proyecto nació de la demanda del alumnado de vincular la teoría con la práctica; de dotar al estudiantado de herramientas pedagógicas y de crear material docente de EpP; de organizar y visibilizar el material didáctico donado por el grupo del Movimiento Cooperativo de la Escuela Popular (MCEP).

Marcamos como objetivos: Generar espacios de experiencia vivencial de EpP; Construir los aprendizajes teóricos de EpP a partir de la vivencia práctica; Dotar al estudiantado de herramientas de reflexión crítica y de técnicas de EpP; Elaborar material didáctico de EpP; Organizar y visibilizar material educativo de MCEP en la Biblioteca de la Facultad de Ciencias de la Educación.

Con el fin de alcanzar los objetivos recurrimos a la metodología endógena, participativa, activa, crítica, necesaria para el desarrollo de la competencia profesional de los futuros educadores para la paz. Aplicamos un método del aprendizaje basado en problemas, fomentando la observación, experimentación y acción colaborativa con ayuda de las técnicas de la Pedagogía de Freinet y de la Educación Emocional-Transpersonal. La práctica y la vivencia personal permitieron construir conceptos epistemológicos propios de la EpP.

La evaluación de la mejora docente fue realizada a través de la evaluación comprensiva, aplicando como instrumentos el informe final del aprendizaje basado en problemas, el Diario de Paz, las sesiones dialogadas.

Como resultado se creó un ambiente de aprendizaje significativo, activo, participativo, afectivo. El alumnado fue iniciado en las técnicas de la escuela democrática, del pensamiento crítico, de la sostenibilidad y del autoconocimiento. Se profundizó en la necesidad de la formación en la EpP. Se inició el proceso de organización y estructuración del material docente para dicha materia. El material educativo del MCEP, depositado en la Biblioteca de la Facultad de Ciencias de la Educación, actualmente está en el proceso de catalogación, digitalización y elaboración de la carpeta virtual $M C E P$ de acceso público.

\footnotetext{
${ }^{1}$ El artículo es resultado del proyecto "Educación para la Paz y Sostenibilidad: un espacio de innovación e intercambio de buenas prácticas docentes", financiado por la Convocatoria de Proyectos de Innovación y Buenas Prácticas Docentes 2018/2020 (PIBD), Universidad de Granada.
} 


\section{Revisión de la literatura}

\subsection{Educación para la paz como construcción disciplinar}

La Educación para la Paz (EpP) es una materia optativa que actualmente se imparte en el Grado de Pedagogía y en el Grado de Educación Infantil. También forma parte del programa del Máster de Paz, Conflictos, Educación y Derechos Humanos y del Máster Universitario Oficial de Profesorado de Educación Secundaria, Formación Profesional, Bachillerato e Idiomas. No obstante, echamos en falta su presencia en la Educación Primaria y en la Educación Social. Desde esta última la EpP podría equipar al estudiantado con instrumentos teóricos, prácticos, técnicos promoviendo, según UNESCO (2019), "la formación y la investigación para el desarrollo sostenible, así como la educación para los derechos humanos, las competencias en materia de relaciones pacíficas, la buena gobernanza, la prevención de conflictos y la consolidación de la paz”. Sáez (2007) recoge EpP en la comunidad disciplinar de Pedagogía Social, con el nombre Educación para la Paz y Ciudadanía, como materia optativa vinculada desde 1991, en la Universidad de Murcia, al área de la Teoría e Historia de la Educación. Próxima a los retos de la Educación Social, dicha materia lleva a la reflexión temas de la exclusión social, género, procesos de inadaptación social, derechos individuales, sociales y de integración, formas de maltrato y violencia, prevención y gestión de los conflictos, protección de la biodiversidad, respeto de la naturaleza. Aunque habría que destacar que la preocupación de EpP supera lo puramente social extendiéndose a un desarrollo integral del ser humano visto desde las dimensiones: Social, Interna y Gaia (Fernández Herrería, 2004, 2015, 2018a, 2019; Smoker y Groff, 1996).

Para que se produzca el desarrollo integral del ser humano, Fernández Herrería y Carmona Orantes (2009) defienden la necesidad de re-hacer la educación, entendiendo que el proceso educativo debería superar una simple trasmisión de los conocimientos, socialización o (a)(en)culturación, y apostar por la madurez personal, por la autorrealización, por el desarrollo pleno de las potencialidades de cada ser humano. Este desarrollo pleno de las potencialidades está en estrecha relación con la prevención de la violencia. Galtung (1995, p. 315) afirma "que la violencia está presente cuando los seres humanos se ven influidos de tal manera que sus realizaciones efectivas, somáticas y mentales, están por debajo de sus realizaciones potenciales”. Así, en el marco de la EpP apostamos por generar espacios de reflexión sobre esta influencia externa e interna, que dificulta la "realización de las potencialidades" del ser humano. Tratamos de crear situaciones de autoconocimiento, autocrítica, autoobservación, el no-juicio, la no-culpa, observación e investigación del contexto, la interrelación con el medioambiente, el respeto a la Comunidad de la Vida. También analizamos las reacciones posibles a distintas situaciones que puedan llevar al conflicto interpersonal. El conocimiento del sujeto de sus reacciones en una situación conflictiva, posibilita intervenir desde la prevención del conflicto en una fase anterior de tener que enfrentarse a su superación o resolución. Interviniendo en una etapa de resolución de conflicto, como puede ser la mediación, incidimos en una conducta de "socorro". El conflicto ya está generado, lo que se busca es que su fuerza sea la menos destructiva para las partes. Sin embargo, si educamos en la cultura de paz, descrita por la ONU (1999) como un estilo de vida basado en el respeto a la vida en todas sus formas, podemos trabajar desde una etapa previa al conflicto (Kárpava, 2019). Según Leonov (2017), el conflicto tiene lugar cuando la situación conflictiva (etapa pre-conflicto) no fue bien direccionada. Cuando se produce el incidente, las partes, que ya 
gestan una sensación de estar en una situación de amenaza directa, viven la intensificación de sus percepciones negativas, lo que desencadena la escalada del conflicto. Desde la Educación podemos dotar a la persona de herramientas para intervenir en la etapa de situación conflictiva (pre-conflicto), donde el incidente todavía no se ha dado y su presencia o ausencia depende de la consciencia de la persona de su forma particular de reaccionar ante él, de su conocimiento de los principios teórico-prácticos que fomentan la cultura de paz y su aplicación práctica. De hecho, Galtung (2016), comparando la Ciencia de la Paz con las Ciencias de la Salud, sugiere actuar desde la Educación con el fin preventivo, creando proyectos, generando tecnologías socio-humanistas que lleven a la población en general a la adquisición de las nuevas competencias en la cultura de paz. Aquí el papel de la Educación es imprescindible para actuar desde el presente sobre la transformación de la visión del mundo de la persona (Cutanda, 2016).

El estudio teórico-empírico de EpP Social, Interna y Gaia es relativamente joven. A finales de los noventa aparece el trabajo de Smoker y Groff (1996) -cuyo comentario y análisis en español se puede consultar en la obra de Fernández Herrería (2015)- donde los autores hablan de seis etapas de desarrollo del estudio de la Paz: 1) Paz entendida como ausencia de guerra entre o dentro de los Estados, 2) como balance de fuerzas en el sistema internacional, 3) introducción por Galtung de los conceptos de Paz Negativa y Paz Positiva, 4) Paz Feminista, que permitió enfocar el estudio de la paz hacia el ámbito "micro". La frontera de lo social se abrió para incorporar la 5) Paz holística Gaia y 6) la Paz holística interna-externa.

Desde el inicio del nuevo milenio Fernández Herrería (2004, 2015, 2018a, 2019) profundiza en el estudio de la Paz Social, Interna y Gaya desde el enfoque pedagógico. Es donde ubicamos nuestra práctica docente e investigadora, y es desde donde enfocamos el Proyecto de la Innovación Docente. No obstante, percibimos que todavía el campo de la Pedagogía para la Paz -vista como práctica investigadora y académica-, así como de la Educación para la Paz -entendida como práctica educativa y profesional-, necesitan profundización en su comprensión y puesta en práctica. Similar a la concepción de la Pedagogía/Educación Social (Sáez, 2007), consideramos necesario pensar la EpP desde los tres campos:

- Campo científico, construyendo la base epistemológica y metodológica desde la investigación desde y para la EpP;

- Campo disciplinar, creando una materia universitaria obligatoria, incorporada en los planes de estudio de todos los grados de Ciencias de la Educación;

- Campo profesional, formando a los educadores para la paz, como actores de transformación de la visión del mundo hacia una mayor aceptación, colaboración, compromiso, obligaciones con uno mismo, con el otro y con la naturaleza, respeto, solidaridad y autorrealización.

La emergente profesión del educador para la Paz se está consolidando desde 1) la Pedagogía para la Paz, creando un campo de conocimiento científico que le da la razón cognitiva; fundando instituciones como, por ejemplo, Instituto de la Paz y los Conflictos; elaborando material formativo en EpP para los programas de grado y postgrado en Ciencias de la Educación; y 2) la Educación para la Paz, cuya intervención tiene carácter práctico, enfocado, por un lado, hacia la prevención de los conflictos, aportando 
herramientas educativas de observación, comprensión y gestión de la autorrealización en la situación conflictiva. En este sentido Jares (2004) define la EpP como,

$$
\begin{aligned}
& \text { un proceso educativo, continuo y permanente, fundamentado en [...] la concepción } \\
& \text { positiva de paz y la perspectiva creativa del conflicto, y que a través de la aplicación } \\
& \text { de métodos problematizantes pretende desarrollar [...] la cultura de la paz, que } \\
& \text { ayude a las personas a desvelar críticamente la realidad desigual, violenta, compleja } \\
& \text { y conflictiva, para poder situarse ante ella y actuar en consecuencia. (p. 34) }
\end{aligned}
$$

Por otro lado, el campo de la acción práctica de la EpP se abre hacia la educación ambiental, educación para la sostenibilidad, educación transpersonal, educación en ecofeminismo y la ética de cuidado, etc.

Históricamente, en España, la EpP ha sido considerada el legado de los movimientos sociales de los grupos noviolentos. La Escuela Nueva y Movimiento de Renovación Pedagógica, por ejemplo, influyeron en que la LOGSE (1990) integrara las cuestiones de EpP como temas transversales del currículo. Sin embargo, según Jares (2000), todavía,

$$
\begin{aligned}
& \text { no contamos con ningún organismo o ministerio que apoye la EpP, como en cambio } \\
& \text { sí tienen la educación no sexista (Instituto de la Mujer), la educación ambiental } \\
& \text { (Ministerio de Medio Ambiente), la educación vial (Dirección General de Tráfico) o } \\
& \text { la educación para la salud (Consejerías y Ministerios de Sanidad). (p. 520) }
\end{aligned}
$$

Centrándonos en la práctica para la Paz, la LEY 27/2005 reconoce el papel decisivo de la educación "como motor de evolución de una sociedad, que pretende [...] sustituir la cultura de la violencia [...] por una cultura de paz" (p. 39418). No obstante, la formación de los profesionales en EpP todavía no ha resuelto el sesgo de concepción negativa de la Paz, que aporta una visión reduccionista a este campo de estudio y de acción. La misma Ley 27/2005 está enfocada en promover técnicas de la paz negativa: resolución de conflicto, construcción de paz en áreas de conflicto, eliminación de todas las formas de discriminación, etc. Los principales temas de interés académico también giran en torno al conflicto, violencia, exclusión social, neoliberalismo, inmigración (Jares, 2000).

La percepción dicotómica del mundo occidental no permite ver la Paz más allá de un concepto utópico, de una gran idea poco tangible. Estamos acostumbrados a la inferencia negativa de la paz, vista como la ausencia de la violencia. Esta idea está en la base del Índice Global de Paz (AAVV, 2018) que mide el nivel de paz de un país a partir de veintitrés indicadores, como el nivel de conflictos internos o externos, el índice de desplazados o la inestabilidad política. Observamos que se miden sólo los aspectos negativos de la paz, sólo lo que no es la paz, o lo que es la paz tras descartar todas las manifestaciones de la violencia (Kárpava, 2015).

Desde nuestra práctica quisiéramos pensar la Paz desde su vertiente positiva, desde la consciencia crítica creadora de la realidad justa, equitativa, sostenible. Desde el desarrollo de las potencialidades del ser humano. Desde una transformación de la percepción del mundo y de la Comunidad de la Vida, a lo que llama la Carta de la Tierra (CT, 2013) en su Preámbulo: "Debemos unirnos para crear una sociedad global sostenible fundada en el respeto hacia la naturaleza, los derechos humanos universales, la justicia económica y una cultura de paz. En torno a este fin, es imperativo que nosotros, los pueblos de la Tierra, declaremos nuestra responsabilidad unos hacia otros, hacia la gran comunidad de la vida y hacia las generaciones futuras". 


\subsection{Tres dimensiones de la educación para la paz: Interna, social y gaia}

Desde el plano Social de EpP se trabaja la educación en ciudadanía, democracia, derechos y responsabilidades de la persona. Desde un modelo crítico-noviolento se generan reflexiones sobre el maltrato, conflicto, violencia, convivencia, exclusión, inmigración, pluri-multi-inter-transculturalidad, diversidad, igualdad, equidad, ciudadanía democrática, derechos humanos (DDHH), etc.

Desde el modelo de educación crítica, inspirada en la Educación Liberadora de Paulo Freire (Freire, 1978, 1990), se apuesta por el desarrollo del hombre inserto-sujeto activo y responsable, dotado de la consciencia crítica, miembro de una sociedad abierta, de la que es agente creador, capaz de tomar consciencia de su rol en la cultura de silencio y dar paso hacia la socio-autorrealización crítica del ser-sujeto. Se apuesta por la persona capaz de transformar el mundo por medio de su acción, capaz de "estar no sólo en el mundo sino con el mundo” (Freire, 1978, p. 28), distanciarse de él, conocerlo, observarse en el mundo y conocerse a sí mismo y actuar desde este conocimiento combinado externo-interno. En este sentido, el educador para la paz, así como el educador social descrito por Freire (1990), tiene que ser flexible para el cambio, tiene que tener la confianza con lo nuevo, conocer sus temores, por ejemplo, el de perder su estatus social en la interacción con los otros, estar abierto a la comunicación, a la reflexión crítica, a la creatividad, a la colaboración, rechazando cualquier vestigio de la manipulación del otro. Un educador para la paz tiene que generar una inquietud en los individuos por el desarrollo de la visión crítica de su realidad.

Es importante apreciar que la realidad social puede transformarse; que está construida por los hombres y que los hombres la pueden cambiar. Es esencial que la concepción ingenua de la realidad dé lugar a una visión capaz de percibirse a sí misma, que el fanatismo sea reemplazado por un optimismo crítico que pueda impulsar a los individuos hacia un compromiso cada vez más crítico con el cambio social radical. [Un educador para la paz social] que opta por el cambio lucha por revelar la realidad. Trabaja "con", nunca "sobre" personas, las que considera sujetos de acción, nunca objetos. [Un educador para la paz social] no teme la libertad, no es manipulador, ni emite prescripciones, rechaza la espontaneidad irreflexiva. (Freire, 1990, pp. 61-62)

El aprendizaje de la Paz Social se construye en base a la teoría crítica, en la comprensión dialéctica de la escolarización como espacio de liberación, que va en contra del adoctrinamiento pasivo de los estudiantes y la conversión en objetos inertes de consumo, rechazando el modelo neoliberal de los espacios educativos, que tienen por prioridad la competencia, excelencia, superioridad, individualismo, poder y egoísmo.

Desde la Educación Crítica se trabaja la responsabilidad y la toma de las decisiones, prácticas asamblearias, escucha activa, participación, cooperación. El trabajo se organiza en forma de tertulias, mesas de discusión, diálogo en torno a la construcción del conocimiento, formando a un educador crítico, capaz de crear un puente entre el conocimiento teórico y práctico. Se favorece el desarrollo del conocimiento emancipador, capaz de analizar los rasgos de la sociedad cerrada y la sociedad en transición (Freire, 1978 ; 1990) con el fin de entender los procesos de dominación, distorsión, manipulación producidos desde el poder. Se apuesta por la consciencia de que la opresión puede ser transformada y superada por medio de la acción liberadora y colectiva.

Desde la perspectiva crítica constructiva se crean los debates y las propuestas de desarticulación de los discursos dominantes como únicos regímenes de verdad, se hacen 
propuestas de búsqueda y de creación de espacios alternativos de aprendizaje que tratan el conflicto como una posibilidad de crecimiento, creatividad, avance y transformación.

\title{
La educación para la paz interna
}

Como habíamos comentado anteriormente, nos basamos en la propuesta de la Paz Social, Interna y Gaya de Fernández Herrería (2015). En el año 1997 Alfonso defendió su Tesis Doctoral bajo título Paz y Educación en J. Krishnamurti, que abrió el camino a la reflexión sobre la Paz Interna en la educación y sobre la poca sensibilidad occidental con la dimensión interna de la paz. En esta obra el autor se acerca al estudio de la Psicología Transpersonal y su aportación a la educación. "Cuando la corriente humanística estaba en período de consolidación y reconocimiento externo, empieza a surgir un nuevo movimiento en Psicología, la llamada cuarta fuerza” (Fernández Herrería, 1997, p. 39), cuyo fin ya no era la autorrealización del yo (fin principal de la Psicología Humanística), sino la trascendencia del yo, "estereotipado y conformado por el medio sociocultural", relacionada con la desidentificación y adquisición de "aquellas experiencias en las que uno progresivamente adquiere consciencia vivencial de su unidad esencial con todos los seres, de un encuentro de uno mismo como una parte de ese todo" (Fernández Herrería, 1997, p. $40)$.

El proceso de la desidentificación lo podemos entender a través del estudio ${ }_{i}$ En la mente o en el Ser? de María Teresa Blázquez Tejada (2015), doctora en Ciencias Físicas. La autora explica la práctica de meditación a través del funcionamiento del sistema nervioso (SN) y la conexión entre los $\mathrm{SN}$ simpático, parasimpático y el SN central superior.

\begin{abstract}
Esta práctica [de meditación] corresponde a una investigación directa de los procesos que tienen lugar en nuestro interior, mediante la observación y la percepción objetivas, es decir, no influenciadas por la manera de pensar. Este desarrollo de la observación y la percepción unido al control sobre los pensamientos produce un aumento de la sensibilidad interior, de la capacidad de percibir, es decir, aumenta la consciencia sobre los procesos que tienen lugar en el interior del cuerpo. En las culturas donde la práctica de la meditación constituía una importante herramienta de investigación se han encontrado escritos que hacen referencia a canales de energía de recorridos similares a los del sistema nervioso. En la tradición india se habla de tres canales de energía, cuya base fisiológica se ha relacionado frecuentemente con el sistema nervioso. Según el Chandoguia-upanishad (texto en sánscrito de mediados del I milenio a. C.) estos canales son llamados nadis y están compuestos por un nadi central llamado susumna, otro izquierdo llamado ida y el derecho llamado pingala (Blázquez Tejada, 2015, p. 158).
\end{abstract}

El proceso de observación interior, que se consigue con la práctica de la meditación, la autora explica a través de cuatro modos básicos de funcionamiento del SN: Pensar, Percibir, Observar y Silencio. La autora propone las siguientes interdependencias:

- Modo Pensar, corteza prefrontal (responsable de las funciones ejecutivas, como hacer planes, tomar decisiones), hemisferio izquierdo² (responsable del aspecto racional, como lenguaje, pensamiento matemático o lógico), ego, futuro. También la zona occipital (responsable de la memoria), hemisferio derecho (capacidad integradora, visual, espacial, no verbal, artística), superego, pasado. Es aquí donde se produce el juicio constante sobre el comportamiento de uno

\footnotetext{
${ }^{2}$ Quisiéramos destacar, que la autora no hace distinción entre el funcionamiento del hemisferio izquierdo y derecho en las personas diestras y zurdas.
} 
mismo y de los demás en relación a los hechos pasados o futuros, sin vivir el presente. Permanecer constantemente en el Modo Pensar aumenta la posibilidad de quedar atrapado en la mente, la que, según Fernández Herrería (1997), deforma nuestra realidad, vinculándola con el dolor, ambiciones, apegos, deseos incumplidos, defensas, obstruyendo la consciencia del Ser.

- Modo Observar: canal central, sistema límbico (comportamiento afectivo, emociones, reconocimiento de objetos nuevos, vigilancia, observación, tratamiento de estímulos olfativos), consciencia del Ser. "El sistema límbico está relacionado $[\ldots]$ con las percepciones interiores. Su desequilibrio suprime la actividad y la atención sostenida” (Blázquez Tejada, 2015, p. 47). Su equilibrio permite tomar distancia con el Modo Pensar y ver de forma objetiva, crítica, las expectativas (ego) y los prejuicios (superego) propios. La "capacidad de observación unida a la plasticidad del cerebro ofrece la posibilidad de realizar cambios en el Modo Pensar" (Blázquez Tejada, 2015, p. 55).

- Modo Percibir: SN central superior, sistema propioceptivo, consciencia corporal (información desde los receptores sensoriales internos y externos, que puede ser debilitada permaneciendo sólo en el Modo Pensar).

- Modo Silencio: en colaboración con los Modos Observar y Percibir se consigue parar el Modo Pensar, conseguir la calma mental, percibir interiormente que el ego individual se despoja por completo de sí mismo. En el proceso de la desidentificación "la consciencia no se identifica con el cuerpo, ni con los sentimientos, ni con las emociones, ni tampoco con los pensamientos" (Fernández Herrería, 1997, p. 49), sino con un estado de liberación, de identificación con la unidad de un todo.

Siguiendo a Fernández Herrería y Carmona Orantes (2009),

reivindicamos la dimensión interna del ser humano, olvidada en el sistema educativo. No es un monopolio de las religiones, sino una dimensión antropológica. El descuido educativo de esta dimensión está provocando una formación de autómatas altamente especializados [...] Necesitamos urgentemente de una cultura interior que nos lleve hacia nuestra identidad más profunda. Vivimos de forma deshumanizada, "perdidos", alienados y "cosificados" en el mundo de las cosas como un objeto más, volcados en la pura exterioridad, incapaces de conectarnos con nuestra realidad interna $[\ldots]$ Esto nos causa una tensión, un estrés y un vacío soterrado [...] que nos lleva al cansancio [...] a la insensibilización, a sentirnos separados [...] La quiebra espiritual se produce cuando todo tiene un precio, pero ya nada tiene valor. (p. 76)

- La Educación para la Paz Gaia viene a ampliar la perspectiva social, otorgada al estudio de la Paz hasta finales del siglo pasado. La amenaza de los desastres medioambientales abrió el camino de la dimensión ecológica en el estudio de la paz. Las Cumbres de la Tierra sobre el cambio climático expresan su preocupación por el futuro inmediato de la civilización humana. El Panel Intergubernamental del Cambio Climático (IPCC) alarma en su último informe sobre las nefastas consecuencias del cambio climático que podríamos presenciar en muy pocos años. El IPCC avisa que tenemos sólo doce años para cambiar nuestra relación con el entorno si queremos prevenir la desaparición de nuestra especie, como muchas otras (Rejón, 2019).

La Teoría de la Paz Gaia integra las dimensiones Social e Interna, incidiendo en la deconstrucción de la visión antropocéntrica del mundo, defendiendo el cuidado del Planeta 
Tierra como el objetivo más importante. Desde la Paz Gaia se retoman las enseñanzas de las culturas ancestrales sobre el cuidado de la Madre Tierra, construyendo el saber en el marco de la Ética del Cuidado, Eco-educación, Ecología Profunda, y Teoría Gaia (Fernández Herrería, 2004, 2015, 2019; Fernández Herrería y Conde, 2010).

Siguiendo la herencia de Fernández Herrería (Fernández Herrería, 2018b; Fernández Herrería y López López, 2010), como herramienta de trabajo, aplicamos la Carta de la Tierra (CT, 2013), una declaración que nace como resultado del acuerdo de una iniciativa de la sociedad civil mundial, defensora de los principios éticos fundamentales para la convivencia democrática, autorrealización, conservación ambiental y el desarrollo sostenible. En su Preámbulo, la CT marca un reto por el cambio de la visión del mundo: "Estamos en un momento crítico de la historia de la Tierra, en el cual la humanidad debe elegir su futuro". Estamos interconectados y somos interdependientes en la trama de la vida con todas las formas de la vida, lo que supone una "responsabilidad universal". "Debemos unirnos para crear una sociedad global sostenible" para cuidar la Tierra y cuidarnos unos a otros "con entendimiento, compasión y amor", "el respeto hacia la Naturaleza, los derechos humanos universales, la justicia económica y una cultura de paz". Debemos tener presente que buscar un "estilo de vida sostenible" significa que "una vez satisfechas las necesidades básicas, el desarrollo humano se refiere primordialmente a ser más, no a tener más”. Nuestros modelos de producción y consumo, lo que consideramos desarrollo, son retos globales que necesitan respuesta para no llegar a la destrucción de las especies, entre ellas la humana. "Se necesitan cambios fundamentales en nuestros valores, instituciones, formas de vida". Para cumplir esto debemos "vivir de acuerdo con un sentido de responsabilidad universal". "Somos ciudadanos de diferentes naciones y de un solo mundo al mismo tiempo" "Necesitamos urgentemente una visión compartida sobre los valores básicos que brinden un fundamento ético para la comunidad mundial emergente" (CT, 2013).

\subsection{Fundamentación normativa de la educación para la paz}

La introducción de la EpP en el ámbito universitario, como un espacio de innovación e intercambio de buenas prácticas docentes, puede ser justificada desde el punto de vista normativo. Haremos una referencia breve sólo a algunos documentos que forman parte de una base mucho más amplia.

1974: en la Conferencia Internacional de Educación, en su 44 ${ }^{a}$ reunión, se propuso la Recomendación sobre la educación para la comprensión, la cooperación y la paz internacionales y la educación relativa a los DDHH y las libertades fundamentales. En este documento los Ministros de Educación se declaran "convencidos de la gran responsabilidad que incumbe no sólo a los padres, sino a la sociedad en su conjunto, de colaborar con todos los actores del sistema educativo y con las organizaciones no gubernamentales a fin de realizar plenamente los objetivos de una EpP, los DDHH y la democracia, y contribuir así al desarrollo sostenible y una cultura de paz" (UNESCO, 1995, Anexo 1, p. 63). Se subraya la importancia del desarrollo pleno de la personalidad del alumno, el respeto de la diversidad cultural, eliminación de todas las discriminaciones, educación en la libertad y dignidad humana, introducción en el proceso educativo de las “estrategias innovadoras adaptadas a las nuevas exigencias de la educación de ciudadanos responsables, comprometidos con la paz, los DDHH, la democracia y el desarrollo sostenible" (UNESCO, 1995, p. 63). Además, en el Anexo II Plan de Acción Integrado sobre la EpP, los DDHH y la Democracia, se hace implícita la recomendación de promover 
"el desarrollo de la paz interior en la mente de los estudiantes para que puedan asentar con mayor firmeza las dotes de tolerancia, solidaridad, voluntad de compartir y atención hacia los demás” (UNESCO, 1995, Anexo 2, p. 65).

1995: las Actas de la Conferencia General de las Naciones Unidas para la Educación, la Ciencia y la Cultura (UNESCO), en el Apartado 5.3, incorporan Proyecto transdisciplinario: Hacia una cultura de paz, invitando a:

5.3.2.A.a) "favorecer la adopción de políticas, planes y programas de educación, y la elaboración y difusión de manuales, libros de texto y material didáctico, de conformidad con el Plan de Acción Integrado sobre la EpP, los DDHH y la Democracia;

5.3.2.A.b) apoyar las redes de instituciones activas en la EpP, los DDHH y la Democracia, haciendo especial hincapié en el fortalecimiento del Plan de Escuelas Asociadas y la red de Cátedras UNESCO;

5.3.2.A.c) promover una producción radiofónica y televisiva encaminada a fomentar el conocimiento y el respeto de culturas [...] y el fomento de actitudes acordes con una cultura de paz;

5.3.2.A.d) efectuar estudio sobre la producción de anuncios publicitarias [...] dirigidos a los niños pequeños, a fin de suscitar en ellos sentimientos opuestos a la violencia" (UNESCO, 1995, p. 60).

1996: Delors pronunció el discurso sobre los objetivos de la educación del nuevo milenio destacando la importancia de aprender a conocer, aprender a hacer, aprender a ser y aprender a estar, que inciden en la necesidad de cambio en la visión del mundo de toda la comunidad educativa (Delors, 1996).

1999: la Asamblea General de las Naciones Unidas emitió la Declaración (53/243) y el Programa de Acción sobre una Cultura de Paz, que afirma: "puesto que las guerras nacen en la mente de los hombres, es en la mente de los hombres donde deben erigirse los baluartes de la paz", "la paz no sólo es la ausencia de conflictos, sino que también requiere un proceso positivo, dinámico y participativo en que se promueva el diálogo y se solucionen los conflictos en un espíritu de entendimiento y cooperación mutuos" (ONU, 1999, pp.1-2).

2005: Ley 27/2005 denuncia la cultura de violencia en la que se basó la educación del siglo XX. Haciendo referencia al manifiesto del Programa de Naciones Unidas para el Desarrollo, de 1988, que denuncia siete inseguridades graves generadores de violencia de tipo económico, rentas, cultural, sanitario, personal, ambiental, político y comunitario, se defiende el papel imprescindible de la educación para promover la cultura de paz. Destaca que corresponde al Gobierno promover la implementación de las asignaturas especializadas en materia de paz, noviolencia, democracia, DDHH, solidaridad, justicia, inclusión; asegurar la promoción de la EpP, como educación a lo largo de la vida; promover centros de investigación universitarios especializados en la materia de paz; concienciar a la población sobre la importancia de la educación en los valores de paz; dotar a la población de técnicas de resolución de conflictos, negociación y mediación, etc.

2006: la Asociación Española para el Desarrollo y la Aplicación del Derecho Internacional de los Derechos Humanos, después de una década de reuniones de los expertos de la UNESCO, firmó el Derecho Humano a la Paz, cuyo Artículo 2 defiende el derecho de toda 
persona a recibir la educación en la paz que "contribuya a generar procesos sociales basados en la confianza, la solidaridad y el respeto mutuo, facilite la solución pacífica de los conflictos y ayude a pensar de una forma nueva las relaciones humanas" (AA.VV, 2008, p.112).

2006: la Ley Orgánica de Educación integra la EpP de forma transdisciplinar y transversal en el currículo académico, destacando que la educación es el medio para garantizar el ejercicio de la ciudadanía democrática, responsable, libre y crítica, indispensable para la constitución de las sociedades abiertas, dinámicas y justas. El Artículo 2 (1.e) destaca la necesidad de

\section{la formación para la paz, el respeto a los DDHH, la vida en común, la cohesión social, la cooperación y solidaridad entre los pueblos, así como la adquisición de valores que propicien el respeto hacia los seres vivos y el medio ambiente [...] y el desarrollo sostenible. (LOE, 2006, p. 17165)}

2013: Ley Orgánica para la Mejora de la Calidad Educativa, aunque sin pronunciarse directamente a favor de la "formación para la paz", hizo alusión a la necesidad de transmisión y puesta en práctica de "valores que favorezcan la libertad personal, la responsabilidad, la ciudadanía democrática, la solidaridad, la tolerancia, la igualdad, el respeto y la justicia, así como aquellos que apuestan por la superación de cualquier tipo de discriminación" (LOMCE, 2013, p.97866).

2011: se redactó la Orden reguladora de la participación de los centros docentes en la Red Andaluza Escuela: Espacio de Paz y el procedimiento para solicitar reconocimiento como Centros Promotores de Convivencia Positiva, la que plantea la necesidad de vinculación de los centros docentes con las asociaciones no gubernamentales, comunidad, familia para colaborar en el desarrollo de una convivencia pacífica, democrática y no violenta (Orden $11 / 04 / 2011)$.

2019: tras veinte años de reflexión sobre la paz en el ámbito académico, el Pleno de la Diputación Provincial de Granada, a petición del Centro de UNESCO de Andalucía y del Instituto de la Paz y los Conflictos, Universidad de Granada, declararon un compromiso de institucionalizar la Cultura de Paz (Diputación de Granada, 2019).

Creemos que es el momento de dar solidez a la EpP como materia independiente que tiene que traspasar una simple discusión sobre aspectos generales de la justicia de paz y estar destinada, según Mayor Zaragoza (2003), a promover la capacidad crítica en las personas, a enseñar técnicas de prevención de los conflictos, en vez de las técnicas paliativas de su resolución, enseñar a responsabilizarse con uno mismo, con el Otro y con el medioambiente y oponerse a todo lo que sea contrario a la vida y a la dignidad humana. Es el momento oportuno para abandonar la cultura de la violencia a favor de la cultura de paz, respeto, responsabilidad, diálogo, participación, escucha, creatividad y autorrealización.

\subsection{Fundamentación psicopedagógica}

Como habíamos comentado en el apartado anterior, la Recomendación sobre la educación para la comprensión, la cooperación y la paz internacionales y la educación relativa a los DDHH y las libertades fundamentales, pronunciada en 1974, hace llamamiento a introducir en el proceso educativo las "estrategias innovadoras adaptadas a las nuevas exigencias de la educación de ciudadanos responsables, comprometidos con la paz, los DDHH, la democracia y el desarrollo sostenible” (UNESCO, 1995, p. 63). La creación de 
estas estrategias necesita un cambio en el enfoque educativo. Para conseguirlo, habíamos creado un equipo interdisciplinar que une los saberes teórico-prácticos de la Pedagogía, Psicología, Irenología, Conflictología, Filología. El aprendizaje fue construido a partir de los principios psicopedagógicos enmarcados dentro de las corrientes humanista, constructivista e intento de aproximación a la corriente transpersonal. En el marco de la Teoría Humanista se intenta desarrollar el aprendizaje significativo, que comprende la interacción de la parte cognitiva con la emocional. Es importante la motivación del alumnado, la libertad en la toma de las decisiones, en la propuesta de los contenidos, formas de trabajo, para que crezca su inquietud y el deseo de descubrimiento propio de la materia a través de la exploración de lo desconocido de forma vivencial. En el marco de la Teoría Constructivista se establecen relaciones entre los conocimientos que ya se tienen y la nueva información, se construyen y se ordenan los conceptos y los contenidos, el alumnado negocia los significados a partir de las experiencias prácticas, propone temas y método de trabajo, lo que genera el contexto motivador. Se trabaja en grupos de forma activa, colaborativa, comprometida, donde se elaboran las estrategias propias de aprendizaje. Desde la corriente transpersonal se trabaja la trascendencia del yo, que va más allá del aprendizaje académico intelectual. La mayor importancia se da a "ser más no a tener más" (CT, 2013), a la quietud, a la observación, al no-juicio.

- Jaenisch y Bird (2003) afirman que el aprendizaje está influenciado por la biología y la experiencia. La genética predispone, pero no determina, aunque es necesario conocerla. La experiencia moldea el cerebro de forma funcional y anatómica. Algunos estudios demostraron como el entrenamiento en mindfulness mejora las funciones ejecutivas en alumnos con Trastornos por Déficit de Atención e Hiperactividad, produciendo cambios desde la interconexión entre circuitos hasta en el balance de neurotransmisores (Semple, 2010).

- El cerebro es un órgano plástico que permite adquirir nuevos aprendizajes a lo largo de la vida (Lenroot y Giedd, 2006). La plasticidad neuronal permite crear nuevos aprendizajes, regenerar áreas funcionales y reestructurar el cerebro. La educación permanente es una forma de entrenar el cerebro, de enriquecer y fortalecer aprendizajes ya realizados. Conforme el alumno aprende, va creando y modificando sus redes neuronales, reforzando las conexiones neuronales más utilizadas, a la vez, desaparecen aquellas redes neuronales que no se activan, debido a la pérdida de su utilidad para la persona. Esto justifica el aprendizaje a lo largo de la vida, aunque es en los primeros años de vida cuando se produce un "superávit de neuronas" que beneficia la creación y modificación de nuevos aprendizajes y como consecuencia, nuevas redes neuronales. Este fenómeno lo conocemos como plasticidad neuronal, según la cual el cerebro es flexible, dinámico y se adapta constantemente a las diversas situaciones. El educador debe tener en cuenta los períodos críticos del aprendizaje y la plasticidad neuronal del cerebro, que afirma que cualquier alumno es capaz de aprender, aunque a un ritmo diferente.

- Cada individuo es único y diferente, por lo que se aconseja trabajar desde un enfoque paidocéntrico. El trabajo con el alumnado requiere la flexibilidad en las actividades para poder responder al ritmo y particularidades individuales de cada alumno. El docente deberá crear sus programaciones didácticas en base al neurodesarrollo de la edad actual del alumno, lo que permitirá un desarrollo progresivo en áreas social, motora, emocional y cognitiva. 
- La cognición tiene que ser trabajada en conjunto con la emoción (Damasio, 1994). La motivación, las metodologías activas, contextualizadas y útiles para la vida, el ambiente positivo, al contrario de las situaciones de estrés, fomentan el aprendizaje significativo. Se le debe dar la importancia necesaria a las emociones dentro del espacio educativo. Es necesario educar a los estudiantes en comprender, interpretar, sentir, gestionar las emociones, tanto propias como ajenas. Esto ayuda a generar el bienestar dentro del aula, así como a potenciar el interés del alumno por la materia (Pintrich, 2003).

- La novedad y la sorpresa activan el sistema de recompensa cerebral. Ante ellas el cerebro se activa, usa diferentes estrategias potenciando el aprendizaje (Howard-Jones y Demetriou, 2009). Este principio justifica el uso del juego como estrategia de aprendizaje eficaz, así como creación de diferentes ambientes de aprendizaje, cambiando constantemente las técnicas y las estrategias educativas, creando sorpresa en el alumnado, motivándolo a descubrir el interés por la exploración de la materia. Este principio llama a potenciar la creatividad, fomentar nuevas técnicas y estrategias educativas, motivar la curiosidad y desarrollar el espíritu investigador del alumno.

- El cuerpo ayuda a entender el mundo, siendo protagonista en el aprendizaje (Carpenter, 2015). La actividad física mejora el aprendizaje potenciando la neurogénesis y la neuroplasticidad (Aberg, 2009). El cuerpo ayuda a entender información abstracta del mundo, por tanto, se deben propiciar aprendizajes activos donde el alumno aprende haciendo, sintiendo y tocando (Carpenter, 2015). Los estudios ponen de manifiesto que inclusión del cuerpo en el proceso de aprendizaje ayuda al alumno a desarrollar conceptos más abstractos y complejos de la realidad. Esto explica la necesidad de introducir las metodologías vinculadas a la práctica del arte o danza que activan las memorias hápticas (táctiles-visuales) y kinestésicas (de movimiento) y mejoran la comprensión del mundo.

- El cerebro del ser humano está provisto de neuronas espejo que explican el aprendizaje por imitación, o el aprendizaje colaborativo basado en casos, problemas o proyectos (Rilling et al., 2002). Las neuronas espejo están altamente implicadas en la formación de la sociedad, en la capacidad empática del ser humano, en su imaginación o la creatividad. Una forma de activar las neuronas espejo es trabajando en grupos, donde se aprende actuar en las situaciones conflictivas y crecer con ellas (Galvis, 2014). Los aprendizajes basados en problemas son idóneos para trabajar la colaboración, toma de decisiones, relaciones democráticas, consenso, responsabilidad y compañerismo.

- El uso del arte potencia las habilidades cognitivo-emocionales. Diversos estudios han demostrado que los alumnos que han sido instruidos con ayuda de educación artística han mejorado su capacidad intelectual. El arte ayuda a potenciar la neuroplasticidad, a crear nuevas redes neuronales que permiten desarrollar una nueva visión del mundo, considerar perspectivas y soluciones distintas a las comunes, ser más creativos y más flexibles.

- La relajación es esencial para el aprendizaje (Spitzer, 2013) porque permite al cerebro consolidar lo aprendido, desechando lo innecesario, activando el proceso 
de fijación en la memoria e integración de los contenidos nuevos con los ya almacenados, lo que justifica el aprendizaje significativo.

- El cerebro tiene un mecanismo de autorregulación cognitiva y emocional que se puede desarrollar (Sheese et al., 2008). Se deben potenciar ambientes educativos multidimensionales que fomenten autogestión del aprendizaje. La flexibilidad cognitiva y una correcta gestión de la inteligencia emocional potencian el pensamiento crítico. La educación emocional proporciona herramientas para actuar ante la adversidad de la realidad, fomentando el éxito de la persona en la vida, su autoconocimiento y respuesta positiva a todo tipo de aprendizaje (Mora, 2013).

\section{Educación para la paz como espacio de innovación docente}

\section{La necesidad detectada}

La idea surgió a partir de la necesidad de combatir la monotonía en la práctica docente tradicional y de romper la brecha que separa la teoría y la práctica. La observación previa mostró que los estudiantes tenían ciertos problemas de interacción, especialmente cuando procedían de grupos distintos; falta de motivación; dependencia de los aparatos tecnológicos; manifestación de tener miedo (al rechazo, al suspenso, a no ser capaz). La adquisición del conocimiento sobre la Cultura de Paz no podía tener un carácter impositivo. Había que crear un ambiente participativo, activo, reflexivo, crítico, comprometido, colaborativo, vivencial, que proporcionara unas herramientas prácticas para la consecutiva construcción teórica del marco de la Paz.

\section{Objetivos}

Se pretendía acercar a los jóvenes a las experiencias del autoconocimiento, autoobservación, facilitando la construcción de una visión del mundo holística, integradora y sistémica. Proporcionar herramientas educativas para el desarrollo del compromiso con la Comunidad de la Vida desde la Educación para la Paz Social, Interna y Gaia. A lo largo del curso se buscaba superar las limitaciones de una visión del mundo antropocéntrica, patriarcal, causa de la crisis global que provocó graves problemas axiológicos, medioambientales, sociales. Se trataba de inspirar el anhelo por el autoconocimiento, por la búsqueda de nuevos horizontes y nuevas visiones de la realidad.

Metodología de trabajo en el aula

- El aprendizaje fue construido a partir de las técnicas de la pedagogía activa, democrática, participativa y flexible, con un especial énfasis en la educación vivencial, axiológica, emocional, inclusiva y colaborativa.

- Partíamos del método del aprendizaje basado en problemas, que permite identificar las necesidades de aprendizaje, buscar la información necesaria para dar respuesta al problema, regresar a él para profundizar en su estudioresolución. Partir de una experiencia práctica para mejorar el proceso cognitivo conlleva riesgos, como intervenir en el proceso tradicional de aprendizaje, cómodo para el estudiantado. Romper con los hábitos de la educación tradicional, aunque criticados por el alumnado, en principio generaba resistencia de los 
estudiantes, que presentaban dificultad para asumir las responsabilidades correspondientes a la nueva forma de aprendizaje-enseñanza de carácter colaborativo.

Algunos autores (VVAA, 2017) previenen sobre las dificultades de partir del problema en una clase familiarizada con el método tradicional. Según ellos, el proceso de integración de una nueva forma de aprendizaje pasa por cinco fases: 1) desconfianza y resistencia; 2) ansiedad y creencia de no avanzar; 3) adquisición de consciencia de ser responsable del aprendizaje propio; 4) seguridad y autosuficiencia; 5) comprensión e integración de la nueva forma de trabajo. Es lo que vivimos durante el proyecto. En la medida en que los estudiantes iban observando sus logros se sentían más seguros, se daban cuenta de las nuevas habilidades adquiridas las que podrían aplicar a otras áreas del conocimiento.

$\mathrm{El}$ aprendizaje basado en problemas facilita trabajo en equipo multidisciplinar, fomenta posibilidad de acceso a la consulta con los expertos de distintas áreas de conocimiento, fortaleciendo y profundizando el trabajo de equipo tanto docente, como estudiantil. Permite no sólo adquirir los conocimientos teóricos, sino incorporarlos a la complejidad de la actuación personal, ciudadana y profesional. Los estudiantes aprendieron a aprender (VVAA, 2017) de forma progresiva e independiente, aplicar los conocimientos a distintas situaciones y problemas, trabajar en equipo, adquirir autonomía, gestionar su tiempo de forma eficaz, identificar los aspectos del problema que ignoran o necesitan explorar en mayor profundidad, investigar por su cuenta, dirigir su propio aprendizaje.

El trabajo se organizó respetando los siguientes pasos:

- El grupo docente propuso problemas prácticos, vividos directamente por los estudiantes de forma participativa y emocional. Mientras los docentes y profesionales externos guiaban el trabajo práctico y coordinaban su teorización, apoyaban en la búsqueda bibliográfica, aconsejando desde el área de su conocimiento, los estudiantes se responsabilizaban de la construcción de su aprendizaje, aplicable a la práctica de la vida diaria.

- A partir de la experiencia vivencial adquirida en los talleres, impartidos en los Seminarios Prácticos, se aclaraban los términos y se discutían los significados básicos de la teoría de la Educación para la Paz, hasta llegar a su comprensión por todos.

- Se definía el problema de la situación experimentada. Se reflexionaba sobre la vivencia. Se trabajaba la escucha activa.

- Se realizaba la lluvia de ideas sobre el problema desde el conocimiento previo de los estudiantes sobre el tema.

- Se sistematizaban y se organizaban las ideas.

- Se formulaban los objetivos del aprendizaje.

- Se realizaba la búsqueda de la información y revisión bibliográfica.

- Se sintetizaba la información, la que posteriormente se reflejaba en el Diario de Paz y en el informe final.

- La intervención práctica con los estudiantes se realizó en tres etapas: 


\section{a) Educación para la paz social}

En esta sección se trataron las cuestiones relativas a la modernidad líquida y la sociedad de miedo "baumantianas"; el individualismo y el colectivismo; el dominio del poder y la libertad; consciencia crítica, democracia y sus formas, ciudadanía; opresor y oprimido; víctima y victimario; conflicto, violencia, no-violencia, paz y su tipología. En los talleres se aplicaron técnicas de la Pedagogía de Freinet: asamblea (como herramienta de prevención y resolución de los conflictos, como práctica de consenso grupal, respeto, turno de palabra, escucha activa, evaluación del trabajo conjunto y final), texto libre (que facilitó desarrollo de la consciencia crítica), investigación del medio (aplicación de la investigación a la vida real), marionetas colaborativas (organización del trabajo grupal, uso del material sostenible, toma de decisiones, creatividad, colaboración, contraste y aceptación de las opiniones de los demás). En esta sección el estudiantado tuvo la oportunidad de establecer contacto con el grupo de maestros de MCEP, participar activamente en las Asambleas, conociendo su tipología, sus fines y su aplicación práctica en el ámbito educativo. También en esta etapa se gestionó la apertura del espacio virtual en la Biblioteca de la Facultad de Ciencias de la Educación que recogió el material educativo donado por el grupo de MCEP.

\section{b) Educación para la paz gaia}

En la segunda etapa se realizó el Taller Educación en la visión del mundo basado en los principios de la Ecoeducación y Ética de Cuidado. Se recurrió a la Declaración de la Carta de la Tierra con el fin de integrar sus principios en la transformación de la realidad del alumnado, promoviendo la Cultura de Paz y Sostenibilidad. En colaboración con la ONG Ávalon Project, iniciativa por la cultura de paz fue organizado el Taller de Storytelling, donde se practicaron técnicas de contar historias tradicionales de distintos países, combinando la narración con actividades creativas y de autoconocimiento. Nos basamos en el estudio de Cutanda (2016) y su última publicación La Colección de Historias de la Tierra (Cutanda, 2019), cuyo objetivo consiste en comunicar la enorme riqueza de los valores trasmitidos por los cuentos tradicionales en relación al cuidado de la comunidad de la vida, así como hacer visible su potencial didáctico-educativo para el desarrollo del pensamiento crítico.

En el mismo marco fue organizada una actividad en la naturaleza El diálogo musical entre la Paz Interna y la Paz Gaia: sonido y conexión con los árboles y los planetas. En un taller al aire libre se vivió una experiencia musical que conectaba el sonido de la guitarra, la expresión del ser humano y respuesta de la naturaleza. El taller fue realizado en dos espacios, con el fin de sentir la diferencia del impacto de la música. Uno cerrado: aula de música de la Facultad, y otro abierto: la montaña. Las vivencias prácticas fueron llevadas a la construcción de la Teoría Gaia y la Sostenibilidad.

\section{c) Educación para la paz interna}

En la tercera etapa, con ayuda de los psicólogos desarrollamos prácticas de la educación emocional, arte, y danza-terapia, atención integral, meditación, relajación llevadas posteriormente a la reflexión sobre los aspectos teóricos de la Paz Interna. A lo largo del taller se trabajó la prevención de los conflictos; el no-juicio; la autoobservación; respuestas de control y liberación de la tensión interna; reconocimiento y comprensión de las emociones, entre ellas las de miedo, ira y tristeza; elección de una opción de reacción a la emoción entre varias posibles. Se ofrecieron herramientas para observar el cuerpo, las emociones, los pensamientos y sus interrelaciones. Se trabajó con la desidentificación con 
el problema y su observación objetiva. El trabajo práctico abrió un diálogo reflexivo sobre el artículo Re-hacer la educación (Fernández Herrería y Carmona Orantes, 2009), cuya comprensión inicial presentaba dificultades a los estudiantes.

\section{Ejemplo de uno de los talleres realizados: Storytelling como técnica de autodescubrimiento}

La técnica Storytelling (Avalon Project, 2019) procede de la práctica ancestral de contar cuentos y trasmitir de forma oral y emotiva la información, los valores, las enseñanzas tansgeneracionales. Esta estrategia, basada en un contacto personal con la historia ancestral y su recreación a través de la expresión artística (verbal, corporal, pictórica, musical), potencia la imaginación, la creatividad, el autoconocimiento, la autocrítica, por lo que puede ser implementada en los diversos contextos educativos, permitiendo transmitir ideas, conceptos, valores, habilidades, directa e indirectamente.

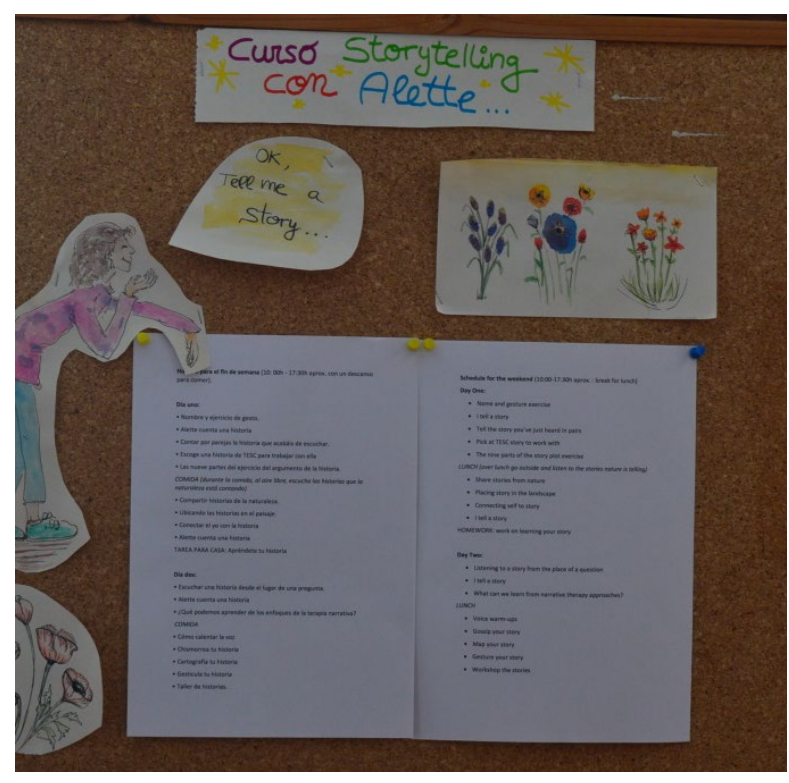

Figura 1. Plan del taller de Storytelling Fuente: Elaboración propia.

El taller fue realizado en dos secciones de ocho horas cada una, en una casa rural -sede de la Asociación Avalon Project- con el fin de proporcionar un espacio de encuentro con uno mismo, con el otro y con la naturaleza. En primer lugar, se hizo una breve introducción en el proyecto The Earth Stories Collection (La Colección de Historias de la Tierra), en la técnica de Storytelling y su uso en la práctica educativa (Cutanda, 2019). Posteriormente se realizaron las actividades propias de esta técnica (figura 1).

Introducción en la narración de los cuentos ancestrales, donde cada uno establece el contacto con una de las historias. En parejas se experimenta la escucha activa y la trasmisión de la historia al otro (figura 2). 


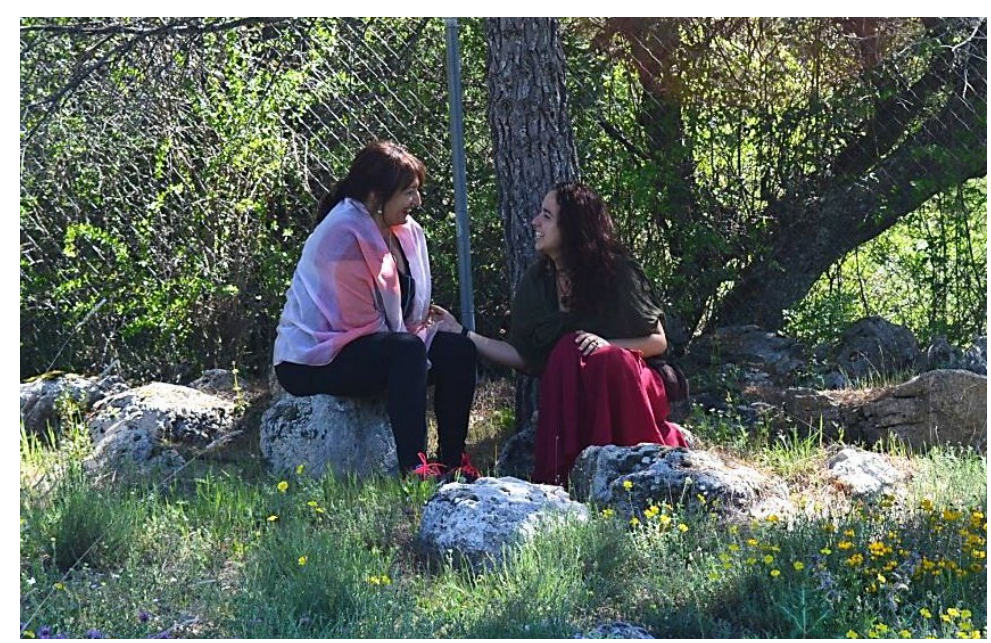

Figura 2. Nos redescubrimos en la historia Fuente: Elaboración propia.

Comprensión del esquema de la historia conlleva una reflexión profunda sobre la "verdad" de la historia y su significado para el narrador y el oyente.

La elaboración del mapa de la historia, con apoyo de las herramientas artísticas, ayuda a entender "por qué me ha elegido la historia que cuento" y escuchar "la respuesta de la historia a mis preguntas existenciales, a mis bloqueos emocionales, a mi forma de ver el mundo".

La explicación del resultado artístico del mapa de la historia ayuda a dar voz a las dudas y los conflictos internos, a las interpretaciones, permite solicitar colaboración, intercambiar ideas, reflexionar sobre la necesidad de transformar las actitudes (figura 3).

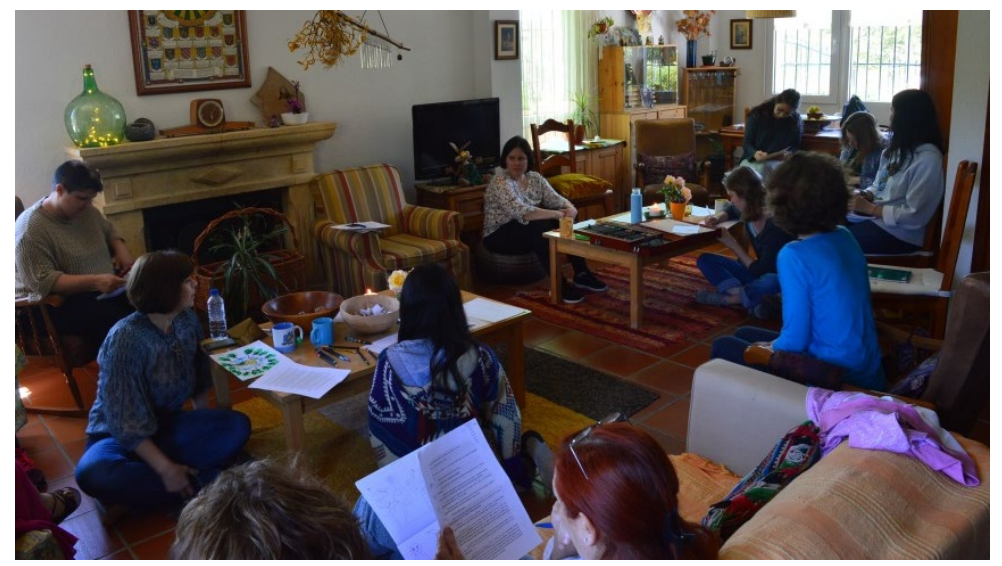

Figura 3. Construyendo la historia en grupo Fuente: Elaboración propia.

En la naturaleza, en contacto con los árboles, se realizan actividades corporales que permiten ver la importancia de cada elemento de la historia y como cambia su función en relación a su ubicación.

Se comparte la experiencia personal de contar cuentos a los terceros. 
En pequeños grupos la historia es contada desde la visión de los distintos personajes (¿Cómo contaría el Lobo su encuentro con la Caperucita Roja?).

Se elige el personaje de la historia y se realiza un diálogo con él. Se le pregunta sobre su pasado, presente y futuro, qué le gusta hacer durante la semana y el fin de la semana, etc. Cualquier pregunta es válida para conocerlo.

Gesticular la historia, sin recurrir a la palabra, ayuda a experimentarla desde nuevas sensaciones.

Tras múltiples vivencias de la misma historia, el acercamiento personal y grupal a su contenido, a los personajes, ideas, prejuicios, conflictos, soluciones, a su estructura, a su verdad, a los detalles, a la palabra, a los movimientos y las quietudes, a los asombros de las vivencias internas y el compartir grupal, a la respuesta de la naturaleza a las preguntas vivenciales, llega la hora de contar la historia al grupo en general. Con esta práctica se produce un aprendizaje armónico de uno mismo, del grupo, de la naturaleza. Un aprendizaje que impulsa la búsqueda de respuestas cognitivas a las preguntas prácticas. Reflexionamos sobre la necesidad de transformar el proceso educativo, sobre la humanización de la educación, el respeto de la Comunidad de la Vida. Buscamos las bases normativas que apoyan esta idea, entre ellas la Carta de la Tierra. Profundizamos en la Teoría de la Ética de Cuidado, Ecofeminismo, Paz Gaia. Los resultados de la búsqueda son llevados a la mesa de discusión y reflejados posteriormente en el Diario de Paz.

\section{Resultados}

Incorporar en el contexto universitario, en los seminarios prácticos de la asignatura Educación para la Paz, las experiencias de meditación, relajación, arte-y danza-terapia, ejercicios corporales enfocados al fomento de la atención integral, prácticas artísticas creativas, provocó sorpresa en el estudiantado, incluso resistencia en el inicio del curso. Esta resistencia, debida al abandono de las técnicas tradicionales de trabajo, criticadas por los estudiantes, pero cómodas por su previsibilidad, hubo que gestionar acomodando el contenido y la práctica de los talleres a las necesidades del estudiantado. A su vez, la sorpresa generaba preguntas que precisaban la búsqueda de información y profundización en el contenido teórico de forma voluntaria. Las dudas se llevaban a las sesiones dialogadas donde se construía no sólo 1) la Teoría de EpP, en su ámbito Social, Interna y Gaia, trabajada desde tres enfoques: normativo, histórico y educativo; sino también 2) la gestión democrática de la interacción grupal, de la toma de decisiones; la libertad de expresión de las emociones, dudas, asombros, hallazgos, desacuerdos; propuesta del plan de trabajo; intercambio con los expertos de forma horizontal; la vivencia directa de la Educación en la Cultura de Paz, que respaldaba el estudio teórico. La propuesta de construir la teoría desde la práctica tuvo buena acogida de los estudiantes, que subrayaron que apostar por la educación como el espacio transformador de la sociedad conlleva, en primer lugar, la transformación de uno mismo, lo que precisa la auto-observación, el auto-conocimiento, el no-juicio, la aceptación, colaboración, creación de la consciencia de grupo, responsabilidad y el actuar desde la escucha y el respeto.

\subsection{Evaluación}

La evaluación de los talleres tenía carácter continuo y comprensivo. Durante el proyecto se realizaron encuentros dialógicos con el fin de intercambiar las impresiones sobre su desarrollo. Por otro lado, fue implementado el Diario de Paz que sirvió para reflejar la 
experiencia del alumnado a lo largo del desarrollo del proyecto, evolución del proceso de la construcción del conocimiento teórico-práctico; búsqueda de la información, reflexión sobre los artículos y las noticias leídas, observaciones, críticas, argumentación, reacciones emocionales, sugerencias y agradecimientos. El Diario permitió mantener un contacto directo con cada estudiante y aportó flexibilidad en el diseño del curso.

Otra herramienta era el informe de aprendizaje basado en problema, que proporcionó información sobre el desarrollo de los aspectos teóricos de la asignatura.

Como resultado final el alumnado respondió positivamente a la nueva organización de la asignatura. Quisiéramos destacar algunos comentarios, recopilados de los Diarios:

Cuando comenzó la clase nos pusimos las sillas formando un círculo, de tal forma que nos pudiéramos ver los unos a los otros. Considero una buena forma de comenzar, ya que así nos podíamos ver las caras de todos y por primera vez no nos dábamos la espalda.

Esta clase me gustó muchísimo, estuvimos tratando la expresión corporal y las emociones. Nos dejamos llevar por nuestro cuerpo con movimientos que nunca podemos hacer en la vida diaria porque 'está mal visto'. La verdad, es que repetiría en otra ocasión.

Me propuse a seguir la lista de las cosas que me hacen feliz: 1. Me encanta llegar a casa y abrazar a mi familia. 2. Sacarle una sonrisa a mis amigos y familia. 3. Explorar el mundo con mi hermana y mis padres.

Estoy contenta con la forma de llevar las clases, ya que pienso que el hecho de que participemos en clase y que realicemos trabajos que tienen relación con la materia nos ayuda no a memorizar las cosas, sino a aprenderlas de una forma más práctica y más dinámica.

Gracias por las técnicas y métodos de aprendizaje que nos han servido para otras asignaturas.

Quisiera destacar la intervención de los invitados de MCEP, „han sido de lo más interesante!

En los comentarios el alumnado subrayaba la necesidad de partir de los problemas reales para dar respuestas concretas a situaciones concretas.

\section{Conclusiones}

Respondiendo a los objetivos marcados, la participación de los estudiantes en el proyecto incrementó su atención, aumentó la motivación y participación en las actividades tanto teóricas, como prácticas. Pero para conseguirlo, hubo que enfrentarse a varias dificultades: temor al fracaso del profesorado y del estudiantado ante nueva forma de trabajo; ruptura con los "cómodos" hábitos de la educación tradicional, resistencia de los estudiantes a nuevo método de trabajo y a ciertas actividades que podrían exponerlos a un juicio externo (y especialmente interno); la dificultad para asumir las responsabilidades correspondientes con la nueva forma de aprendizaje-enseñanza de carácter colaborativo, etc. No obstante, mientras se desarrollaba el proyecto, la horizontalidad entre profesorado-alumnado, la apertura hacia la construcción del conocimiento en común, el respeto mutuo, la confianza permitieron a los estudiantes mostrarse cada vez más flexibles, perdiendo miedo a expresarse, eran más activos, reflexivos, inquietos, interesados por el descubrimiento y construcción de su aprendizaje. Partir de las herramientas prácticas transdisciplinares permitió construir un conocimiento teórico más sólido sobre la materia de la Educación para la Paz, ampliando unos y "perdiendo" otros contenidos. El contacto con los expertos 
permitió acercar el discurso académico a la acción práctica. La relación con los maestros del MCEP dio a la palabra abstracta sobre la educación democrática un contenido experiencial. Hay que reconocer, que quedan muchos aspectos para trabajar, por lo que se decidió continuar con la idea durante el curso académico 2019-2020. La experiencia práctica reflexionada será presentada al terminar esta nueva fase del proyecto.

\section{Referencias}

AA.VV. (2018). Global peace index: Measuring peace in a complex world. Institute for Economics \& Peace.

AA.VV. (2017). El aprendizaje basado en problemas como técnica didáctica. Instituto Tecnológico y de Estudios Superiores de Monterrey

AA.VV. (2008). Declaración de Luarca sobre el derecho humano a la paz. Revista de Paz y Conflictos, $1,109-119$.

Aberg, M. (2009). Cardiovascular fitness is associated with cognition in young adulthood. PNAS, 106(49), 20906-20911. https://doi.org/10.1073/pnas.0905307106

Avalon Project. (2019). The earth stories collection. ONG Avalon Project.

Blázquez Tejada, M. T. (2015). ¿En la mente o en el ser? Ruiz de Aloza Editores.

Carpenter, S. (2015). Cognición corporizada. Mente y Cerebro, 73, 16-21.

CT. (2013). La carta de la tierra en acción: La iniciativa de la carta de la tierra. http://www.earthcharterjordan.org/esp/about.html.

Cutanda, A. (2016). Relatos tradicionales y carta de la tierra: Hacia una educación en la visión del mundo sistémico-compleja (Tesis doctoral). Universidad de Granada, España.

Cutanda, A. (2019). The earth stories collection. Como hacer otro mundo posible con mitos, leyendas y relatos tradicionales. Asociación Avalon Project.

Damasio A. (1994). Descartes' error: Emotions, reason, and the human brain. Avon.

Delors, J. (1996). La educación encierra un tesoro. Santillana.

Fernández Herrería, A. (1997). Paz y educación en J. Krishnamurti (Tesis doctoral). Universidad de Granada, España.

Fernández Herrería, A. (2004). Voces: Paz gaia, paz interna, paz social, ecología profunda, educación para la paz, enfoque humanístico-transpersonal. En M. López Martínez (Dir.), Enciclopedia de paz y conflictos (pp. 37-45) Universidad de Granada.

Fernández Herrería, A. (2005). Mundo emocional y silencio a través de la observación lúcida. En VVAA, Libro de Comunicaciones V Congreso Internacional de Filosofía de la Educación (pp. 391400). Dykinson.

Fernández Herrería, A. (2015). Teoría de la paz. Evolución del concepto de paz. Una propuesta de reconstrucción. En A. Soriano Díaz y M. Bedmar Moreno (Coords.), Temas de pedagogía social-educación social, (pp. 89-132). Universidad de Granada.

Fernández Herrería, A. (2018a). Educación para la paz y la sostenibilidad. Experiencias de reconexión con la comunidad de la vida. En C. Guadalupe García, C. (Coord.), Ética aplicada. Experiencias en educación universitaria (pp. 11-53). Torres Asociados.

Fernández Herrería, A. (2018b). La Carta de la tierra y la educación. Cuadernos de Pedagogía, 485, $72-76$. 
Fernández Herrería, A. (2019). La conexión con la comunidad de la vida. Una estrategia didáctica desde la interiorización. En D. Velazquez y H. Vargas (Coords.), Educación en consumo ético y agroecológico. Hacia una soberanía alimentaria procomunidad de la vida, (pp. 104-132). Dykinson.

Fernández Herrería, A. y Carmona Orantes, G. (2009). Re-hacer la educación: Los mapas del desarrollo humano. Teoría de Educación, 21(2), 45-78.

Fernández Herrería, A. y Conde Caveda, J. L. (2010). La ecopedagogía en la formación inicial de maestros. Investigación en la Escuela, 71, 39-49.

Fernández Herrería, A y López López, M. C. (2010). La educación en valores desde la carta de la tierra. Por una pedagogía del cuidado. Revista Iberoamericana de Educación, 53(4), 1-19.

Freire, P. (1978). Educación como práctica de la libertad. Siglo XXI Editores.

Freire, P. (1990). La naturaleza política de la educación. Cultura, poder y liberación. Paidós.

Galtung, J. (1995). Investigaciones teóricas. Sociedad y cultura contemporáneas. Tecnos.

Galtung, J. (2016). Educación para la paz: Desafío de nuestros tiempos. ONU.

Galvis, R. I. (2014). Las neuronas espejo y el desarrollo de la empatía frente a la agresión y el conflicto en la escuela. Praxis Pedagógica, 15, 43-53. https://doi.org/10.26620/uniminuto.praxis.14.15.2014.43-53

Howard-Jones P. y Demetriou S. (2009). Uncertainty and engagement with learning games. Instructional Science, 37, 519-536. https://doi.org/10.1007/s 1 1251-008-9073-6

Jaenisch R. y Bird A. (2003). Epigenetic regulation of gene expression: How the genome integrates intrinsic and environmental signals. Nature Genetics, 33, 245-254. https://doi.org/10.1038/ng1089

Jares, X. (2000). La educación para la paz en el umbral del nuevo siglo: retos y necesidades. En X. Martínez (Ed.), Materiales del seminario de investigación para la paz (pp. 509-536). Ediciones de la Universidad de Zaragoza.

Jares, X. (2004). Educar para la paz en tiempos difíciles. Bakeaz.

Kárpava, A. (2015). Paz como objeto del conocimiento científico.Conflictología, Revista CientíficoPráctica, 3, 81-96.

Kárpava, A. (2019). Educación para la paz: Paso previo a la mediación. VVAA. (Eds.), Actas del IV Jornada académica da ADESA Direito Internacional e Relações Internacionais. Universidade Estácio de Sá.

Lenroot, R. K y Giedd, J. N. (2006). Brain development in children and adolescents: Insights from anatomical magnetic resonance imaging. Neuroscience \& Biobehavioral Reviews, 30(6), 718 729. https://doi.org/10.1016/j.neubiorev.2006.06.001

Leonov, N. (2017). Bases de la Conflictología, Eirene.

Mayor Zaragoza, F. (2003). Educación para la paz. Educación XXI, 7(1), 17-24. https://doi.org/10.5944/educxx 1.6.0.350

Mora, F. (2013). Neuroeducación. Solo se puede aprender aquello que se ama. Alianza.

ONU. (1999). Declaración 53/243. Programa de acción sobre una cultura de paz. ONU.

Pintrich, P. (2003). A motivational science perspective on the role of student motivation in learning and teaching contexts. Journal of Educational Psychology, 95(4), 667-686.

https://doi.org/10.1037/0022-0663.95.4.667 
Rejón, R. (20 de marzo de 2019). La ONU alerta de una catástrofe ambiental en 2050: Millones de muertes por contaminación y devastación en el Ártico, El Diario. es.

https://www.eldiario.es/sociedad/ONU-alerta-catastrofe-ambiental_o_877362829.html

Rilling, J., Gutman, D., Zeh, T., Pagnoni, G., Berns, G. y Kilts, C. (2002). A neural basis for social cooperation. Neuron, 35(2), 395-405. https://doi.org/10.1016/So896-6273(02)00755-9

Sáez Carreras, J. (2007). Pedagogía social y educación social. Historia, profesión y competencias. Pearson.

Semple, R. (2010). Does Mindfulness meditation enhance attention? A randomized controlled trial. Mindfulness, 1, 121-130. https://doi.org/10.1007/s12671-010-0017-2

Sheese, B., Rothbart, M., Posner, M., White, L. y Fraundorf, S. (2008). Executive attention and self-regulation in infancy. Infant Behavior and Development, 31, 501-510. https://doi.org/10.1016/j.infbeh.2008.02.001

Smoker, P. y Groff, L. (1996). Creating global-local cultures of peace. Peace and Conflict Studies, 3(1), art. 3.

Spitzer, M. (2013). Demencia digital: El peligro de las nuevas tecnologías. Ediciones B.

UNESCO. (1995). Proyecto transdisciplinario: Hacia una cultura de paz. UNESCO.

UNESCO. (2019). Cultura de paz y no violencia. UNESCO.

\section{Breve CV de las autoras}

\section{Alena Kárpava}

Doctora en Paz, Conflictos y Democracia, Universidad de Granada. Personal docente investigador del Departamento de Pedagogía de la Facultad de Ciencias de la Educación y del Deporte, campus de Melilla, Universidad de Granada, España. Miembro colaborador del Instituto de la Paz y los Conflictos, UGR. Miembro del Grupo de Investigación Valores emergentes, Educación Social y Políticas Educativas (HUM 580), UGR. Vocal de Educación del Centro UNESCO de Andalucía. Asesora en el tema de Educación para la Paz en la ONG Proyecto Ávalon- Iniciativa para una Cultura de Paz. Líneas de investigación: Irenología, Educación para la Paz, Paz Intercultural, conflicto comunicacional, Educación Social. Tiene publicados en torno de 50 trabajos académicos (libros, capítulos de libros y artículos científicos). ORCID ID: https://orcid.org/o0o00003-4887-2361. Email: akarpava@ugr.es

\section{Verónica Juárez Ramos}

Doctora en Neurociencia cognitiva y del comportamiento, Universidad de Granada. Personal docente investigador del Departamento de Psicología Evolutiva y de la Educación, Facultad de Formación de Profesorado de la Universidad de Extremadura. Miembro del Grupo de Investigación Entrenamiento Cognitivo de la Universidad de Granada (SEJ 497). Líneas de investigación: El proceso de toma de decisiones desde la Neurociencia Cognitiva, Sesgos Cognitivos en toma de decisiones, Neurociencia Educativa, Inteligencia Emocional y aprendizaje activo. Tiene 30 publicaciones científicas con artículos académicos en revistas indexadas en JCR y Scopus. Además, tiene publicado un libro y capítulos de libro en editoriales internacionales y nacionales de prestigio demostrado. ORCID: https://orcid.org/o000-0003-4329-9463.Email: vjuarez@unex.es 